Meta

Journal des tradlucteurs

Translators' Journal

\title{
Les longueurs de la traduction
}

\section{Louis Kokas}

Volume 14, numéro 2, juin 1969

URI : https://id.erudit.org/iderudit/003334ar

DOI : https://doi.org/10.7202/003334ar

Aller au sommaire du numéro

Éditeur(s)

Les Presses de l'Université de Montréal

ISSN

0026-0452 (imprimé)

1492-1421 (numérique)

Découvrir la revue

Citer cet article

Kokas, L. (1969). Les longueurs de la traduction. Meta, 14(2), 93-97.

https://doi.org/10.7202/003334ar

Ce document est protégé par la loi sur le droit d'auteur. L'utilisation des services d'Érudit (y compris la reproduction) est assujettie à sa politique d'utilisation que vous pouvez consulter en ligne.

https://apropos.erudit.org/fr/usagers/politique-dutilisation/
Cet article est diffusé et préservé par Érudit.

Érudit est un consortium interuniversitaire sans but lucratif composé de l’Université de Montréal, l'Université Laval et l'Université du Québec à Montréal. Il a pour mission la promotion et la valorisation de la recherche. https://www.erudit.org/fr/ 


\section{les longueurs de la traduction}

Des relevés statistiques semblent prouver qu'en général un texte est plus long dans la langue d'arrivée que dans la langue de départ. Le traducteur est instinctivement porté à allonger, d'abord par prudence, puis par ignorance et voire même par hâte, sous la pression des délais qui lui sont imposés. Les mots de Pline le Jeune nous reviennent à l'esprit: «... ma lettre est longue, parce que cette fois-ci je n'ai pas le temps d'écrire en peu de mots... ». En outre, il y a de nombreux cas où la clarté exige amplification, dilution.

Cette observation générale vaut-elle aussi pour la version vers l'anglais? Hilaire Belloc, qu'on aime citer quand il s'agit de traduction, affirma: « Une traduction doit forcément être plus longue que l'original... Ceci vaut en premier lieu pour les traductions de n'importe quelle langue vers l'anglais. Puisque l'anglais n'a pas tant de désinences à sa disposition que la plupart des langues, nous sommes constamment contraints d'exprimer des choses, par l'addition d'une particule, qui dans d'autres langues sont déjà contenues dans la forme du mot. »

Or, si une traduction par exemple du français vers l'anglais devient plus longue, les traductions de l'anglais vers le français devraient nécessairement devenir plus courtes. Or, c'est précisément du contraire dont on nous parle toujours. Belloc avait-il tort?

Les raisons qu'on donne pour expliquer le fait que la version est plus longue que le thème, sont variées. Les traducteurs expérimentés soulignent qu'en anglais les monosyllabes sont très fréquents, qu'on ne peut souvent rendre des propositions participiales que par des propositions conjonctives, et finalement qu'en général, une phrase anglaise est bien profilée, comme si elle avait été construite en application rigoureuse des principes aérodynamiques (streamlined serait en effet une traduction plus courte). Déjà, Jacob Grimm, fondateur de la philologie allemande, a dû concéder qu'en ce qui concerne la concision, aucune langue moderne n'est à même d'égaler l'anglais, "pas même notre langue allemande ». 
En effet, dans quelle autre langue pourrait-on dire en aussi peu de mots que Joe est le propriétaire d'un établissement ouvert toute la nuit et où on vend des saucisses, comme je l'ai vu sur une enseigne à New York: Joe's All Night Sausages.

La longueur de la version peut être aussi due à une sorte d'illusion d'optique, une sorte de scrupule professionnel de la part du traducteur, qui, fasciné par la teneur de l'original, s'y accroche comme saisi par une crampe et croit lui rester redevable s'il ne traduit pas chaque mot particulier par un autre mot. Pourtant, le rapport entre l'original et la version n'est pas le même qu'entre les deux membres d'une équation algébrique.

On sait aussi que l'anglais aime se servir de mots images tandis que d'autres langues emploient des termes exprimant des notions abstraites, donc des mots signes. Or, les notions abstraites sont tirées le plus souvent de racines grecques ou latines et probablement plus longues que l'image correspondante qui est exprimée en anglais par un mot image monosyllabique. Quelques exemples: pile atomique surgénératrice, breeder; numération globulaire, blood count; pilotage sans visibilité, blind flying; affectation de patriotisme, flag waving; prix de présence, door price; bibliophile, book lover; exposition canine, dog show; période de prospérité économique, boom; etc.

Il n'est donc point étonnant que la version devienne plus volumineuse, si l'on tient compte aussi du fait général qu'un mot à signification multiple ne peut que rarement être traduit également par un seul mot d'une autre langue.

À ces arguments, sans doute déjà valables aux temps de Hilaire Belloc, d'autres s'ajoutent, de plus récente date.

En ce qui concerne le vocabulaire, la langue anglaise, telle qu'on s'en sert sur ce continent dans les quotidiens, dans les périodiques, dans la littérature, à la télévision et à la radio, a évolué beaucoup depuis le temps de Belloc. On tient davantage compte du conseil de Fowler: se servir de préférence de mots courts au lieu de mots longs et de mots germaniques au lieu de mots d'origine romane. Or, le français se méfie de mots trop courts, dit-on; on pourrait ajouter: et aussi des néologismes. Par contre, l'américain démontre souvent une méfiance saine envers des idées qui ne peuvent être exposées à l'aide des expressions de langue familière. Une évolution plus marquée se dessine donc dans l'usage des différents niveaux de la langue, une forte tendance au relâchement linguistique, à l'usage des locutions cmpruntécs à la langue parlée. Le Oxford English Dictionary illustre les différents niveaux de la langue par le schéma suivant:

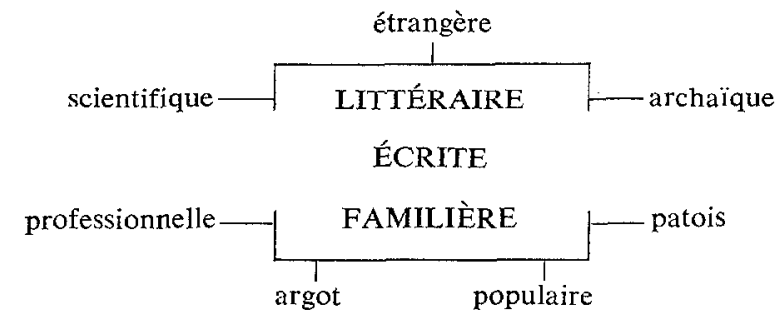

Or, il semble que la langue écrite subit un assaut, une poussée, de la part de la langue familière, renforcée par la multitude des néologismes et des auxiliaires venant de la langue scientifique, professionnelle, populaire, de l'argot et des 
langues étrangères. Dorénavant, la langue familière semble partager le centre avec la langue écrite proprement dite. Ce schéma statique pourrait être complété par un deuxième, un schéma dynamique, reflétant l'évolution récente:

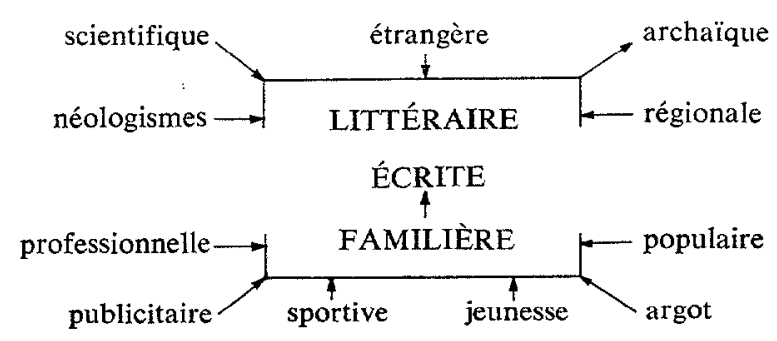

Une tendance similaire, mais qui est loin d'avoir une telle ampleur, existe aussi en français, et de même qu'en allemand, mais d'une importance minime. On peut également constater qu'en anglais écrit les préoccupations esthétiques semblent être de plus en plus reléguées au second plan par les préoccupations fonctionnelles comme brièveté, simplicité, popularité, valeur émotive et évocatrice, etc., ce qui se traduit surtout par des transformations lexicales. Par contre, en français, la différence entre la langue familière et la langue écrite est moins marquée et se manifeste non pas tant par le choix des mots et par le souci de propriété des termes que par des déviations syntaxiques.

Le résultat de cette pénétration en profondeur entraîne un déplacement de la langue écrite anglaise qui fait qu'elle ne coïncide plus avec la langue écrite française ou allemande. L'image doit être filtrée ou brisée et reflétée à un angle différent à l'instar des rayons de lumière tombant sur la surface de l'eau; la musique doit être transposée d'un mode dans un autre, de majeur en mineur, du forte au piano, etc., selon le cas. Je cite ce passage d'un article paru dans un quotidien de Montréal: «Says Dr. Wood [Dr. W. Donald Wood, Professor of Economics and Director of the Industrial Relations Centre at Queen's University]: While realizing it is difficult to prescribe a detailed, precise framework, one does not have to be an intellectual giant to recognize when income and price adjustments are completely outside the economic ball-park. » $\mathrm{Si}$ on ne s'attend pas à trouver des termes sportifs dans les constatations savantes d'un économiste français qui se respecte, c'est parfaitement bon style «langue écrite» en anglais de l'Amérique du Nord. Ce qui nous intéresse le plus ici, c'est que dans la version française, il serait recommandable de remplacer l'image empruntée à la langue des sports par des expressions empruntées à la langue scientifique, ce qui rendra presque toujours la version plus longue que le thème.

"Outside the economic ball-park». De la part d'un économiste français distingué, on s'attendrait probablement à une expression d'un autre niveau de langue, par exemple: «En dehors du champ d'application des doctrines économiques». La manchette d'un autre quotidien de Montréal nous apprend que: «Stanfield puts muzzle on Camp $»$. On lit dans les journaux que les hauts fonctionnaires du gouvernement fédéral et les leaders du syndicat cherchent "to iron out the bugs ». En français pareilles images seraient déplacées. En France, on men- 
tionnerait probablement que «M. Camp a été condamné au silence par M. Stanfield » et qu'on est en train « d'aplanir les difficultés ». L'Américain s'affaire souvent en pullover, en bras de chemise ou en pyjama où l'Européen met une tenue plus soignée. Une traduction plus longue en résulte dans bien des cas.

Cette énumération des facteurs, auxquels on peut attribuer le fait que la traduction est souvent plus longue que l'original anglais, ne serait complète si on ne mentionnait pas les difficultés dues à la langue de la publicité en Amérique du Nord. Celle-ci constitue un niveau sui generis et n'a guère de pendant dans d'autres langues. Le plus souvent on se trouve dans la nécessité de la rendre par la langue écrite. Seulement, dans la traduction d'un texte publicitaire ou commercial souvent un espace strictement égal est attribué aux différentes langues. En effet, la langue publicitaire française ne s'inspire ni de la psychologie ni du style de la publicité américaine, de sorte qu'il incombe de transposer le message publicitaire en français au lieu de le traduire. Entre parenthèses, j'ai la conviction que la publicité, pour être vraiment efficace, devrait être conçue dans la langue du public auquel elle est destinée, la traduction ne devrait donc même pas entrer en ligne de compte.

Finalement, une expérience personnelle qui en dit beaucoup sur la concision de la langue anglaise parlée. Dans une charcuterie allemande de Montréal, un jour je fus témoin d'une conversation entre le patron (d'origine allemande) et un visiteur, d'origine allemande lui aussi. Après avoir écouté pendant quelque temps les propos de son interlocuteur qui écorchait l'anglais de son mieux, le patron l'encouragea: «Parlez donc l'allemand, je le comprends très bien!» Le visiteur changea donc de langue, d'abord soulagé, puis ralentit graduellement, visiblement gêné. Tout à coup, il éclata: "Listen, I have no time to speak in German 》 et continua sa tirade dans son anglais à lui.

Se peut-il que quelque chose ait échappé à Hilaire Belloc ? Peut-être a-t-il fermé les yeux sur le fait que ce qui vaut pour une langue vaut aussi pour une autre?

Il est évident que le traducteur se voit souvent contraint de faire un travail diffus parce qu'un idiotisme n'a pas de locution réciproque dans la langue de la version. Est-ce qu'il n'arrive pas aussi souvent que nous puissions rendre un message, pour lequel une langue étrangère a besoin de plusieurs mots, avec un seul?

Est-ce que le traducteur, en resserrant adroitement l'énoncé, ne peut pas s'approcher de la concision de l'original ? MM. Vinay et Darbelnet consacrent dans leur Stylistique comparée tout un chapitre à l'économie et traitent des différents moyens qui permettent de réaliser cette économie stylistique si nécessaire. Pourrait-on supposer que l'emploi de ces outils, ces moyens d'économie dans les deux langues joue aussi souvent en faveur du traducteur que contre lui ? L'opportunité d'employer en français peu de mots sans sacrifier quelque chose du message, se présente de temps en temps. Il importe de bien comprendre l'énoncé et d'être, pour ainsi dire, à l'affût des économies. N'est-on pas quitte envers l'auteur (James Boswell) si on traduit because he had gone into taverns and ordered meals which he could not pay for par «pour grivèlerie » et wegen Zechprellerei ? Au lieu de dire «Société royale pour la prévention de la cruauté envers 
les animaux (R.S.P.C.A.)» ne suffit-il pas de dire «Société protectrice des animaux 》 et Tierschutzverein, pour engineering shop, «atelier 》 et Werkstatt, pour cut away deadwood, «émonder » et säubern, pour blind, indiscriminate admiration, de dire « idolâtrie » et Verhimmelung, et pour organization pattern, de dire «plan» et Aufstellung?

Aussi, nombreux sont en anglais les clichés, les locutions, les unités de traduction qui, selon la mentalité française, sont des pléonasmes. Les textes juridiques en sont pleins. Rules and regulations, body corporate and politic, terms and conditions, trade-in allowance, fine and dandy, comfort and convenience, ways and means se rendent en français presque toujours par un seul mot. On pourrait aussi dépister des pléonasmes dissimulés, par exemple dans le signe No parking at any time. Le bon traducteur peut donner toute sa mesure dans des raccourcis. Mais, gare à vous! Il y a là des pièges! C'est dans un piège d'équivoque que l'infortuné traducteur est tombé, en rendant sur une affiche: Support Expo 67, par « Supportez l'Expo 67 ».

LOUIS KoKAS 